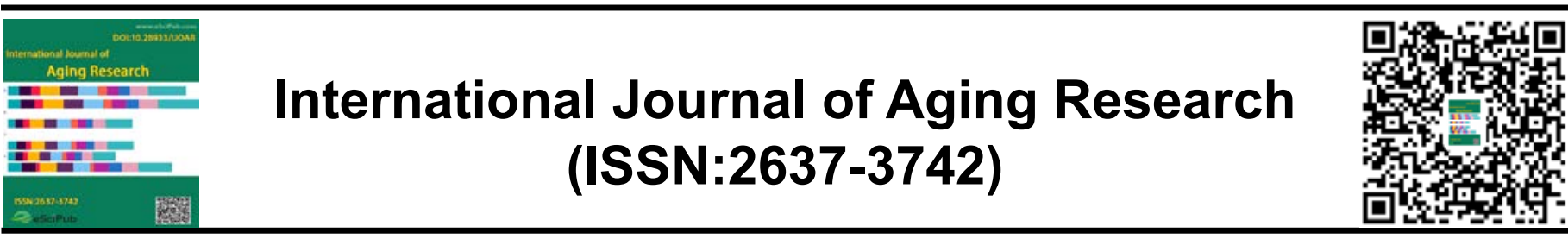

\title{
Prevalence of Polypharmacy and Association to Pharmacotherapy Complexity in Older HIV-Positive Patients. The Sevihlla Study
}

${ }^{1}$ Ramón Morillo-Verdugo. PharmD, ${ }^{1} \mathrm{M}^{\mathrm{a}}$ de las Aguas Robustillo-Cortés*. PharmD., ${ }^{2}$ Laila Abdel-Kader Martín. PharmD., ${ }^{3}$ María Álvarez de Sotomayor Paz. PhD., ${ }^{4}$ Fernando Lozano de León Naranjo. MD, ${ }^{5}$ Carmen Victoria Almeida-González. MS

${ }^{1}$ Valme Hospital (Pharmacy Department). Seville, 41014, Spain.

${ }^{2}$ Virgen del Rocío Hospital (Pharmacy Department), Seville, 41013, Spain.

${ }^{3}$ School of Pharmacy. University of Seville.

${ }^{4}$ Unit of Infectious Diseases and Microbiology, Valme Hospital, Seville, 41014, Spain.

${ }^{5}$ University of Seville.

\begin{abstract}
Background: Increased life expectancy of older HIV-positive patients has been associated to a parallel increase in age-related comorbidities.
\end{abstract}

Objectives: To ascertain the prevalence of polypharmacy and its association to pharmacotherapy complexity, as measured by the Medication Regimen Complexity Index, in older HIV-positive patients; to calculate the median value of pharmacotherapy complexity; to identify polypharmacy and multimorbidity patterns; and to address adherence to antiretroviral and concomitant drugs.

Methods: A cross-sectional, observational study was conducted in patients over 50 years of age receiving active antiretroviral drugs during 2014 at outpatient pharmacy services of a tertiary hospital in Spain. Data collected from the electronic medical record included demographic, clinical and comorbidity related endpoints.

The primary endpoint was the proportion of patients with polypharmacy and major polypharmacy. Polypharmacy was defined as treatment with six or more drugs (including antiretroviral). Major polypharmacy (more than 11 drugs) was also considered. Patients was categorized according to their polypharmacy pattern. Three patterns were applied based on age of participants: cardiovascular, depression-anxiety, and chronic obstructive pulmonary (COPD) disease patterns. A patient was classified into
${ }^{*}$ Correspondence to Author:

$M^{a}$ de las Aguas Robustillo Cortés. Pharmacy Department, Valme Hospital, av Bellavista s/n 41014, Seville, Spain.

How to cite this article:

Ramón Morillo-Verdugo, $\mathrm{M}^{\mathrm{a}}$ de las Aguas Robustillo-Cortés, Laila Abdel-Kader Martín, María Álvarez de Sotomayor Paz, Fernando Lozano de León Naranjo, Carmen Victoria Almeida-González.Prevalence of Polypharmacy and Association to Pharmacotherapy Complexity in Older HIV-Positive Patients. The Sevihlla Study. International Journal of Aging Research, 2019, 2:35

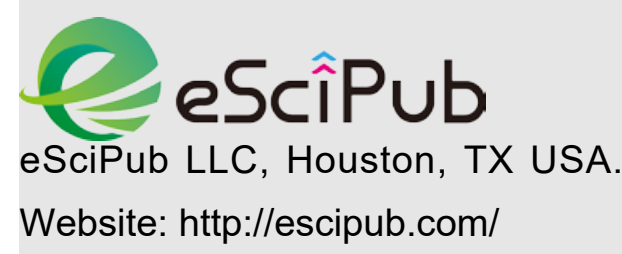


a pattern when at least three drugs of the treatment were in the same pattern.

Antiretroviral treatment adherence was measured using the SMAQ questionnaire and hospital dispensing records. Adherence to concomitant medication was measured using the Morisky-Green questionnaire and electronic pharmacy dispensing records.

Pharmacotherapy complexity index, as assessed by $\mathrm{MRCl}$, was also considered. Patients were classified as low MRCI (less than 14 points) or high $\mathrm{MRCI}$ (more than 14 points).

Results: The study sample consisted of 223 patients ( $86.5 \%$ men), with a median age of 53.0 years. More than $80.0 \%$ of the patients were viro-inmunological controlled. Prevalence of polypharmacy was $56.1 \%$. The median value of pharmacotherapy complexity was 11.0 . The main contribution to this value was from the concomitant medication.

The polypharmacy pattern mainly calculated was cardiovascular (60.0\%) and the multimorbidity pattern was cardiometabolic (73.8\%).

Presence of polypharmacy was associated to greater pharmacotherapy complexity $(p<0,001)$. Adequate adherence to the antiretroviral and to concomitant medication was found in $83.6 \%$ and $37.9 \%$ of patients respectively.

Conclusions: More than a half of the older HIV-positive patients received six or more different drugs with a significant pharmacotherapy complexity showing adequate adherence to antiretroviral but not to concomitant drugs. Cardiovascular conditions were most common in terms of prescriptions and comorbidities.

Keywords: HIV, Polypharmacy, Pharmacotherapy Complexity, Aging

\section{INTRODUCTION}

Worldwide mortality related to the acquired immunodeficiency syndrome (AIDS) of people infected by the human immunodeficiency virus (HIV) has decreased over the years, largely as the result of introduction of highly active antiretroviral therapy (ART). Thus, HIV-infected individuals live longer. It is estimated that by 2030 nearly three-quarters of people living with HIV will be 50 years or older. Age-related conditions such as cardiovascular disease, kidney disease, and non-AIDS-defining cancers are likely to continue to increase among HIVinfected patients as their median age also increases ${ }^{1,2}$. This high prevalence of comorbidities has only exacerbated the polypharmacy problem, which has recently become a clinical concern among providers caring for HIV-infected patients ${ }^{3-5}$.

There are different definitions of polypharmacy. In numerical terms, it is most commonly defined as at least five or more prescription drugs, 
which is also associated to worse health outcomes in older patients ${ }^{6}$. Polypharmacy should be considered the next challenge in clinical follow-up of HIV-infected patients for several reasons. First, HIV-positive patients are taking increasingly more drugs to manage multimorbidity ${ }^{7}$. Second, polypharmacy has a major impact on traditional aspects such as adherence, leading to worse treatment outcomes ${ }^{8-12}$. Third, the risk of clinically relevant drug interactions increases with polypharmacy, and the aging HIV population is at an even higher risk ${ }^{13}$.

Finally, another critical but less known factor is pharmacotherapy complexity (PC). Martin et al. developed a method for quantifying antiretroviral regimen complexity for HIV patients ${ }^{14}$. This method was the first step toward obtaining a better understanding of the impact of complex ART regimens on adherence and clinical outcomes. Libby et al. subsequently developed a medication regimen complexity index (MRCl) to estimate complexity of all drugs taken by a patient (HIV and non-HIV drugs) ${ }^{15}$. Although the impact of regimen complexity, number of drugs, and pill burden on medication adherence has been addressed in scientific literature, most studies have focused on use of antiretrovirals and have failed to consider the impact of other drugs or therapies. No studies addressing this issue have been published yet.

\section{Study objective}

The purpose of this study was to determine the prevalence of polypharmacy and its association to $\mathrm{PC}$, as measured by $\mathrm{MRCl}$, in older HIVpositive patients. The secondary objectives were to calculate the median value of PC; to identify polypharmacy and multimorbidity patterns, describing the concomitant medication most often prescribed and assessing the PC index, with attention to the appearance of drugs (pharmaceutical form, dosage, and specific instructions); and to address adherence to antiretroviral and concomitant drugs.
A final objective was to explore whether the complexity index is associated to adherence, based on the multimorbidity pattern.

\section{Ethics approval}

The study was approved by the institutional ethics committee of the South Seville area. Participants were given written information about the study and its objectives, and those who agreed to take part provided their written informed consent.

\section{MATERIALS AND METHODS}

A cross-sectional, observational study was conducted. Patients enrolled in the study met the following inclusion criteria: HIV patients over 50 years of age on active ART drugs from 1 January 2014 to 31 December 2014. Participants were given written information about the study and its objectives, and those who agreed to participate provided their written informed consent.

Patients participating in another clinical trial or who did not sign the informed consent were excluded.

Data collected from the electronic medical record included demographic data (sex and age) and HIV transmission mode; clinical endpoints: plasma viral load (copies/milliliter $[\mathrm{mL}]$ ) and CD4+ T-cell count (cells/microliter), and comorbidity related endpoints (number and type of comorbidities, particularly liver, central nervous system and cardiovascular disease or high blood pressure). Charlson comorbidity index was also included ${ }^{16}$.

Pharmacotherapy endpoints included ART regimen and concomitant medications (drugs for acid-related disorders, psycholeptics or psychoanaleptics, lipid-lowering drugs and other drugs for cardiovascular disease or diabetes, among others). The PC index, as assessed by $\mathrm{MRCl}$, was also considered. This validated tool includes 65 items divided into three subgroups: dose forms, dosing frequencies, and additional instructions relevant to drug administration. The calculated value was obtained through the web tool of Colorado 
University

available

http://www.ucdenver.edu/academics/colleges/p harmacy/Research/researchareas/Pages/MRC Tool.aspx) ${ }^{17}$. Based on a previous study, patients were classified, by consensus definition, as low $\mathrm{MRCl}$ (less than 14 points) or high $\mathrm{MRCl}$ (more than14 points) ${ }^{15}$.

ART adherence was measured using the SMAQ questionnaire and hospital dispensing records. Adherence to concomitant medication was measured using the Morisky-Green questionnaire and electronic pharmacy dispensing records.

The independent variable was polypharmacy, defined as treatment with six or more drugs (including antiretroviral therapy). Major polypharmacy (more than 11 drugs) and excessive polypharmacy (more than 21 drugs) were also considered.

Polypharmacy pattern was analyzed according to the Calderón Larrañaga et al study ${ }^{18}$, with a non-random association in drug prescription resulting in polypharmacy patterns. Three patterns were applied based on age of participants: cardiovascular, depression-anxiety, and chronic obstructive pulmonary (COPD) disease patterns, with a different prevalence between men and women. A patient was classified into a pattern when at least three drugs of the treatment were in the same pattern. To calculate the corresponding polypharmacy patterns of each patient, drugs were classified according to the Anatomical Therapeutic Chemical Classification System (ATC) using only the first three levels of the classification.

Finally, multimorbidity patterns was analyzed according to the Prados-Torres et al study. Chronic diseases resulted in three multimorbidity patterns: cardiometabolic, depressive-psychogeriatric, and mechanicalthyroidal ${ }^{19}$. Patients were classified into a type if they had two diseases included in a pattern.ART drugs were obtained from a pharmacy-dispensing outpatient program
(Dominion-Farmatools()). Non-ART drugs prescribed were provided by an electronic health prescription program of the Andalusian Public Health System. The remaining endpoints were obtained from laboratory tests, microbiology reports, and from the review of the medical history of each patient.

\section{Statistical analyses}

Quantitative variables were given as mean and standard deviation or as median and interquartile range (IQR) in case of a skewed distribution. Qualitative variables were given as percentages (\%).

To compare mean values of quantitative variables between groups, a Student's t test was used for independent samples, or a MannWhitney non-parametric $U$ test in case of nonnormal distributions. If significant differences were seen, 95\% confidence intervals were found for differences in mean (or median, if relevant) values. Contingency tables were prepared and a Chi-square test was used to analyze the relationship between qualitative variables; otherwise, the Monte Carlo simulation with asymptotic method and exact test was used.

Pearson correlation coefficients were calculated, and independence tests were performed to analyze the association between total PC, ART complexity, physicianconcomitant index, and Charlson index. Data were analyzed using IBM SPSS Statistics version 22.0 software

\section{RESULTS}

A total of 223 patients with a median age of 53.0 years (IQR: 52.0-57.0), 86.5\% males, were enrolled into the study. Baseline demographic, clinical, and lifestyle characteristics of patients are shown in Table 1.

Median comorbidities per patient were 3.0 (IQR: 2.0-4.0) with a median Charlson index of 2.4 (IQR: 2.0-3.2). Viral liver diseases were diagnosed in $67.3 \%$ of patients, cardiovascular diseases or HBP in $25 \%$, and central nervous system diseases in $20.5 \%$ of patients. 
Table 1. Baseline demographic, clinical, and lifestyle characteristics of patients

\begin{tabular}{|c|c|c|}
\hline \multicolumn{2}{|l|}{ Characteristics ( $\mathrm{n}=223$ patients) } & \\
\hline \multicolumn{3}{|l|}{ Demographic parameters } \\
\hline \multicolumn{2}{|l|}{ Sex (male) n (\%) } & $153(86.5)$ \\
\hline \multicolumn{2}{|l|}{ Age (years) (median + IQR) } & $53.0(52.0-57.0)$ \\
\hline \multirow{3}{*}{$\begin{array}{l}\text { HIV risk factor } \\
\text { n (\%) }\end{array}$} & IDU & $121(54.3)$ \\
\hline & Sexual & $68(30.5)$ \\
\hline & Unknown & $34(15.2)$ \\
\hline \multicolumn{3}{|l|}{ Clinical parameters } \\
\hline \multicolumn{2}{|l|}{ Undetectable plasma viral load (<50 copies $/ \mathrm{mL}$ ) n(\%) } & $184(84.4)$ \\
\hline \multirow{2}{*}{$\begin{array}{l}\text { CD4 levels } \\
n(\%)\end{array}$} & $<200$ cells $/ \mu \mathrm{L}$ & $22(10)$ \\
\hline & $\geq 200$ cells $/ \mu \mathrm{L}$ & $198(90)$ \\
\hline \multicolumn{3}{|l|}{ LIFESTYLE } \\
\hline \multicolumn{2}{|l|}{ BMI (kg/m2) (median+lQR) } & $24.6(22.2-27.6)$ \\
\hline \multicolumn{2}{|l|}{ Smoking $(n=149) n(\%)$} & $66(44.3)$ \\
\hline \multicolumn{2}{|l|}{ Alcohol consumption $(n=156) n(\%)$} & $42(26.9)$ \\
\hline \multicolumn{2}{|l|}{ Active drug users $(n=153) n(\%)$} & $10(6.5)$ \\
\hline \multicolumn{2}{|l|}{ Patients with controlled BP $(n=53) n(\%)$} & $47(88.7)$ \\
\hline \multirow{3}{*}{$\begin{array}{l}\text { History of heart disease, HBP or diabetes mellitus } \\
(n=188) n(\%)\end{array}$} & Yes & $15(8.1)$ \\
\hline & No & $87(46.2)$ \\
\hline & Not known & $86(45.7)$ \\
\hline \multicolumn{2}{|l|}{ Physical activity $(n=31) n(\%)$} & $13(41.9)$ \\
\hline
\end{tabular}

IQR, interquartile range; IDU, injection drug users; BMI, body mass index; HBP, high blood pressure;

\section{Polypharmacy Pattern}

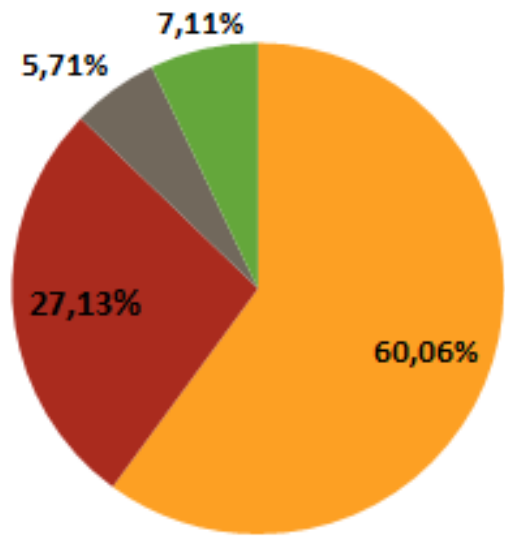

Cardiovascular

Depression-anxiety

- COPD

Mixed

Figure 1. Polypharmacy pattern. COPD: chronic obstructive pulmonary disease 


\section{Multi-Morbidity Pattern}

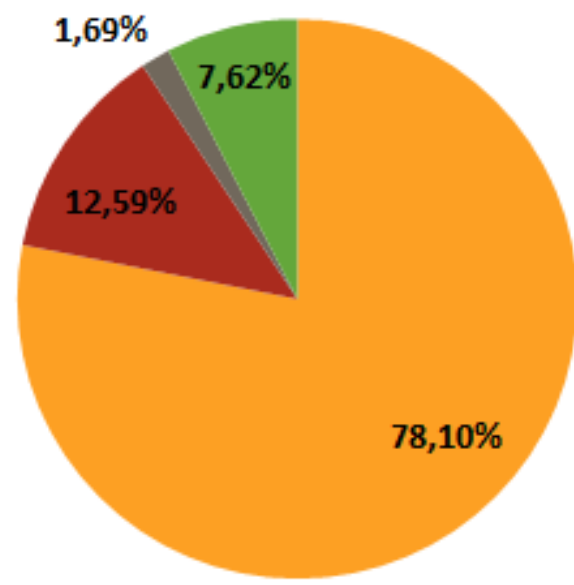

Cardio-metabolic

Depressive-psychogeriatric

nechanical-thyroidal

mixed

Figure 2. Multi-morbidity pattern

ART regimens consisted of two nucleoside reverse transcriptase inhibitors (NRTI) plus a non-nucleoside reverse transcriptase inhibitor (NNRTI) in $37.2 \%$ of patients; two NRTIs plus a boosted protease inhibitor in 18.8\%; two NRTIs plus an integrase strand transfer inhibitor (INSTI) in $12.6 \%$, and other combinations in $31.4 \%$ of patients. A majority (52\%) of patients started antiretroviral therapy before 2002, and $14.8 \%$ had been on three or more ART regimens. The median number of concomitant drugs prescribed was 3.0 (1.0-5.0). The most commonly prescribed therapeutic drug classes included psychotropic drugs (35.9\%), lipidlowering drugs $(29.1 \%)$, cardiovascular agents (29.1\%), drugs for gastroesophageal reflux disease $(26.9 \%)$, and glucose-lowering drugs $(11.7 \%)$.

The proportion of patients with high PC was $40.4 \%$, with a median overall score of 11 (IQR:7-18).

Adequate adherence to ART and concomitant medication was found in $83.6 \%$ and $37.9 \%$ of patients respectively.

As regards the main variables, $56.1 \%$ of patients had polypharmacy, major polypharmacy in $9.4 \%$ of cases. Of the 70 patients who were calculated the polypharmacy pattern, $60.0 \%$ were cardiovascular, and of the 126 patients with multimorbidity pattern, $73.8 \%$ were cardiometabolic (Figs. 1 and 2).

Presence of polypharmacy was associated to higher PC values. Patients with high PC indices had a 50 times higher chance $(p=0.0001)$ of polypharmacy than those with low PC values. The PC index and the concomitant medication index significantly correlated with the three index rating sections (pharmaceutical form, dosage, and instructions). The ART complexity index was only related to dosage and instructions $(p<0.05)$.

As regards the influence of the polypharmacy pattern on PC, the concomitant and overall PC index score was different $(p<0.05)$ in patients with cardiovascular pattern, polypharmacy pattern score for COPD and mixed, which also occurred in the case of the depression-anxiety pattern. However, no significant differences were found between the cardiovascular and depression-anxiety patterns.

Finally, the influence of overall PC on adherence was analyzed depending on the polypharmacy and metabolic pattern presented by the patients. PC did not affect adherence to both patterns. However, among patients who had the COPD or the mixed multimorbidity 
pattern, those with high complexity had a $20 \%$ increase in adherence.

PC did not affect adherence to both patterns. However, among patients who had a COPD or mixed multimorbidity pattern, those with high PC had a $20 \%$ greater adherence level.

\section{DISCUSSION}

Our study shows that half the elderly HIVpositive patients currently have polypharmacy. These data are consistent with those reported by other authors $8,13,20$.

The available literature includes different definitions of polypharmacy. Use of five drugs has been a widely accepted criterion to define polypharmacy, but we suggest use of six drugs based on the most recent recommendations ${ }^{6}$. The proportion of patients on polypharmacy in our study was however similar to that reported by other studies. The number of patients with major polypharmacy in our study $(9.4 \%)$ is particularly important. According to the literature, polypharmacy will increase in the coming years ${ }^{10}$. Because of this and the progressive physiological deterioration of patients, in addition to the classical assessment of actual or potential drug interactions, it will become increasingly common and necessary to use other terminology about use of drugs in other types of chronic patients, such as potentially inappropriate medication or cholinergic risk.

The value of PC in our study was 11.0. As expected, concomitant drugs represented the main contribution to the PC value. There are multiple definitions of polypharmacy. Some of them include use of at least one unnecessary medication and different thresholds for the number of drugs in a patient's regimen ${ }^{21-23}$. There is a clear relationship between harm and polypharmacy. Those who are on 5-6 drugs have a lower risk than those on 7-8 or 9 drugs 24-26.In a retrospective HIV-positive cohort, Sutton et al. ${ }^{27}$ analyzed a different concept, the pill burden, which was associated to adherence and risk of hospitalization. Authors concluded that single tablet regimens (STR) were associated to higher adherence rates and lower hospitalization risks. A recent retrospective study found an association between polypharmacy, and a lower probability of using STR 28. Currently available STRs may be limited in this aging population by complex drug-drug and drug-disease interactions, and the desire to make treatment regimens more flexible.

Our results are similar to those of Zhou et al ${ }^{29}$, who showed that older age, comorbidity, and longer duration of HIV infection were associated to high pill burden. However, the study included HIV-infected patients given care at an outpatient clinic, but not all of them were over 50 years of age.

Designing ART regimens that do not interact with other chronic medications or exacerbate comorbidities may be challenging, especially in heavily pretreated patients whose ART options are limited. However, these results also suggest that use of PC scores adds information, extending beyond a simple pill count or pill burden concept.

Greater prescription and filling complexity is known to be associated to lower adherence in cardiovascular patients. To date, all studies have shown that polypharmacy is robustly associated to non-adherence, but no study has focused on the HIV population in a large cohort in real practice. Non-adherence to ART is especially worrying because it is associated to decreased viral suppression, allows for viral resistance, and increases the risk of opportunistic diseases ${ }^{30-32}$.

A systematic review in non-HIV-infected patients has shown that although variability was seen in the association between complexity and adherence, most studies concluded that an increased regimen complexity decreases medication adherence ${ }^{33}$. In our study, the overall adherence calculated was high for ART, but particularly low for concomitant medication, where the main polypharmacy pattern was precisely cardiovascular. This suggests 
prioritization of drug intake by patients based on their beliefs and perceptions about drugs ${ }^{34}$. The results of the Borrego et al. ${ }^{35}$ study showed that adherence problems begin at the time of prescription, because one third of patients will not even collect the prescribed medication.

These results suggest that, in older HIV patients, all prescribed drugs should be checked at least every six months in individuals prescribed more than four drugs, and at least once a year in all others. Clinical guidelines recommend systematic review of the prescribed drug therapy following a sequential and structured methodology ${ }^{6}$.

Our results suggest that PC is associated to cardiovascular polypharmacy and depressionanxiety patterns. As expected, the complexity index was only related to dosage and instructions, since tablets are the most common pharmaceutical form currently available. As reported, dosing frequency made the largest contribution of all components $(62 \%)$ to the PC ${ }^{36}$. In this line of research, these types of factors should thoroughly be reviewed when assessing prescriptions and the daily management plan of patients, especially for most commonly prescribed drugs.

We propose a redefinition of the concept of polypharmacy to include not only the quantitative aspect of the number of prescribed drugs but, above all, regimen complexity. A cutoff PC value needs to be proposed to determine if a patient meets the polypharmacy criterion.

\section{Limitations}

A common limitation of other published studies

is that they only include data on officially prescribed drugs, thus excluding private health system treatments or alternative medicines. However, this is not seen as a very significant limitation in our study; because of the universal coverage of the public health system in Spain, only a small number of patients use alternative medications. However, the $\mathrm{MRCl}$ is an imperfect tool and faces tradeoffs between sensitivity and specificity, as most clinical measures do. Despite a long list of possible dose formulations, frequencies, and directions, some options are missed, such as once monthly, and details such as how to code two once-daily drugs that cannot be taken at the same time are not considered. There are other possibilities to analyze PC, such as the ARCl, including many more such details. As the average complexity score is not significantly different from the $\mathrm{MRCl}$ for ART regimens, we prefer the $\mathrm{MRCl}$ because it has been more widely studied in the literature in recent years $15,37,38$

It is known that multimorbidity contributes to further vulnerability and complexity in clinical management in the contemporary ART age. Interest in methods to identify individuals at risk of multimorbidity is strongest. In geriatric medicine, such multisystem vulnerability is quantified using the construct of frailty, which is a state of increased vulnerability related to degradation of homeostatic mechanisms ${ }^{39}$. The concept of frailty may be useful for discriminating whether morbidities themselves or the toxicity of prescribed treatments make a greater contribution to adverse outcomes ${ }^{40}$.

Because of the changing face of the HIV epidemic, healthcare providers will be increasingly challenged to effectively manage older HIV-infected patients with multimorbidity, polypharmacy, and high PC. It is important to increase our understanding of polypharmacy in the ever increasing number of older HIVinfected patients in order to be able to develop prevention strategies for the problems inherent to old age and multiple treatments.

Future research efforts will focus on risk assessment, such as that offered by the PC Index, to inform the prioritization of medications based on their risks and benefits for each patient. In addition, efforts should be made to promote public health and multidisciplinary initiatives, behavioral changes, and prevention aimed at reducing polypharmacy and PC. 
The PC index may prove to be useful as a risk prediction tool. Additional studies are needed to establish its power and to reveal potential opportunities for clinical intervention to decrease medication complexity as a risk factor for nonadherence. Decreasing dosing frequencies, simplification of administration routes, or counseling on non-prescribed supplements and its consequences in use of health resources, including hospitalizations, are proposed.

\section{CONCLUSION}

In conclusion, more than a half of older HIVpositive patients in this study received six or more different drugs, with a significant PC. Cardiovascular conditions and depressionanxiety were most common in terms of prescriptions and comorbidities. The concept of polypharmacy should include not only the number of prescribed drugs, but also their complexity.

\section{Conflicts of interest}

The authors report no financial conflicts of interest related to the subjects discussed in this article.

\section{Financial support}

This project received no funding.

\section{REFERENCES}

1. Tseng A, Szadkowski L, Walmsley S, Salit I, Raboud J. Association of Age With Polypharmacy and Risk of Drug Interactions With Antiretroviral Medications in HIV-Positive Patients. Ann

Pharmacother. 2013;47(11):1429-1439. doi:10.1177/1060028013504075.

2. High KP, Brennan-Ing M, Clifford DB, et al. HIV and aging: state of knowledge and areas of critical need for research. A report to the $\mathrm{NIH}$ Office of AIDS Research by the HIV and Aging Working Group. J Acquir Immune Defic Syndr. 2012;60 Suppl 1:S1-18. doi:10.1097/QAI.0b013e31825a3668.

3. Gleason LJ, Luque AE, Shah K. Polypharmacy in the HIV-infected older adult population. Clin Interv Aging. 2013;8:749-763. doi:10.2147/CIA.S37738.

4. Gnjidic D, Hilmer SN, Blyth FM, et al.
Polypharmacy cutoff and outcomes: five or more medicines were used to identify community-dwelling older men at risk of different adverse outcomes. J Clin Epidemiol. 2012;65(9):989-995.

doi:10.1016/j.jclinepi.2012.02.018.

5. Gimeno-Gracia M, Crusells-Canales MJ, Rabanaque-Hernández MJ. Clinical characteristics and antiretroviral treatment of older HIV-infected patients. Int J Clin Pharm. 2014;36(6):1190-1195. doi:10.1007/s11096014-0015-x.

6. Grupo de expertos de la Secretaría del Plan Nacional sobre el SIDA (SPNS), Sociedad Española de Geriatría y Gerontología (SEGG). Documento de consenso sobre edad avanzada e infección por el virus de la inmunodeficiencia 2015; (Noviembre).

humana.

7. Smith JM, Flexner C. The challenge of polypharmacy in an aging population and implications for future antiretroviral therapy development. AIDS. 2017;31 Suppl 2:S173S184. doi:10.1097/QAD.0000000000001401.

8. Gimeno-Gracia M, Crusells-Canales MJ, Javier Armesto-Gómez F, RabanaqueHernández MJ. Prevalence of concomitant medications in older $\mathrm{HIV}_{+}$patients and comparison with general population. HIV Clin Trials. 2015;16(3):117-124. doi:10.1179/1528433614Z.0000000012.

9. Cantudo-Cuenca MR, Jiménez-Galán R, Almeida-González C V., Morillo-Verdugo R. Concurrent Use of Comedications Reduces Adherence to Antiretroviral Therapy Among HIV-Infected Patients. J Manag Care Pharm. 2014;20(8):844-850. doi:10.18553/jmcp.2014.20.8.844.

10. Smit $M$, Brinkman $K$, Geerlings $S$, et al. Future challenges for clinical care of an ageing population infected with HIV: a modelling study. Lancet Infect Dis. 2015;15(7):810-818. doi:10.1016/S14733099(15)00056-0.

11. Marzolini C, Elzi L, Gibbons S, et al. Prevalence of comedications and effect of potential drug-drug interactions in the Swiss HIV Cohort Study. Antivir Ther. 2010;15(3):413-423. doi:10.3851/IMP1540.

12. Izquierdo YB, Agudo PM, Ramírez ÁA, Labriola $\mathrm{Cl}$, González CA, Verdugo RM. Influence of co-medication on the risk of clinically relevant drug interactions in patients with HIV. Eur J Hosp Pharm. 2013;20(2):88- 
Ramón Morillo-Verdugo et al., IJOAR, 2019 2:35

92. doi:10.1136/ejhpharm-2012-000249.

13. Holtzman C, Armon C, Tedaldi E, et al. Polypharmacy and Risk of Antiretroviral Drug Interactions Among the Aging HIV-Infected Population. $J$ Gen Intern Med. 2013;28(10):1302-1310. doi:10.1007/s11606013-2449-6.

14. Martin S, Wolters PL, Calabrese SK, et al. The Antiretroviral Regimen Complexity Index. A novel method of quantifying regimen complexity. J Acquir Immune Defic Syndr. 2007;45(5):535-544.

doi:10.1097/QAI.0b013e31811ed1f1.

15. Libby AM, Fish DN, Hosokawa PW, et al. Patient-level medication regimen complexity across populations with chronic disease. Clin Ther. 2013;35(4):385-398.e1. doi:10.1016/j.clinthera.2013.02.019.

16. Charlson ME, Pompei $\mathrm{P}$, Ales $\mathrm{KL}$, MacKenzie CR. A new method of classifying prognostic comorbidity in longitudinal studies: development and validation. J Chronic Dis. 1987;40(5):373-383.

http://www.ncbi.nlm.nih.gov/pubmed/3558716 . Accessed January 24, 2018.

17. Universidad de Colorado. Patient-level Medication Regimen Complexity in Defined Clinical Populations. http://www.ucdenver.edu/academics/colleges/ pharmacy/Research/researchareas/Pages/M RCTool.aspx. Accessed September 19, 2017.

18. Calderón-Larrañaga A, Gimeno-Feliu LA, González-Rubio F, et al. Polypharmacy patterns: unravelling systematic associations between prescribed medications. Carvajal A, ed. PLoS One. 2013;8(12):e84967. doi:10.1371/journal.pone.0084967.

19. Prados-Torres A, Poblador-Plou B, CalderónLarrañaga $A$, et al. Multimorbidity Patterns in Primary Care: Interactions among Chronic Diseases Using Factor Analysis. Gagnier JJ, ed. PLoS One. 2012;7(2):e32190. doi:10.1371/journal.pone.0032190.

20. Edelman EJ, Gordon KS, Glover J, McNicholl IR, Fiellin DA, Justice AC. The next therapeutic challenge in HIV: polypharmacy. Drugs Aging. 2013;30(8):613-628. doi:10.1007/s40266-013-0093-9.

21. Shah BM, Hajjar ER. Polypharmacy, Adverse Drug Reactions, and Geriatric Syndromes. Clin Geriatr Med. 2012;28(2):173-186. doi:10.1016/j.cger.2012.01.002.

22. Salazar JA, Poon I, Nair M. Clinical consequences of polypharmacy in elderly: expect the unexpected, think the unthinkable. Expert Opin Drug Saf. 2007;6(6):695-704. doi:10.1517/14740338.6.6.695.

23. Colley CA, Lucas LM. Polypharmacy: the cure becomes the disease. J Gen Intern Med. 1993;8(5):278-283.

http://www.ncbi.nlm.nih.gov/pubmed/8505690 . Accessed January 24, 2018.

24. Jyrkkä J, Enlund H, Lavikainen P, Sulkava R, Hartikainen S. Association of polypharmacy with nutritional status, functional ability and cognitive capacity over a three-year period in an elderly population. Pharmacoepidemiol Drug Saf. 2011;20(5):514-522. doi:10.1002/pds.2116.

25. Gandhi TK, Weingart SN, Borus J, et al. Adverse Drug Events in Ambulatory Care. N Engl J Med. 2003;348(16):1556-1564. doi:10.1056/NEJMsa020703.

26. Jyrkkä J, Enlund H, Korhonen MJ, Sulkava R, Hartikainen S. Polypharmacy status as an indicator of mortality in an elderly population. Drugs Aging. 2009;26(12):1039-1048. doi:10.2165/11319530-000000000-00000.

27. Scott Sutton S, Magagnoli J, Hardin JW. Impact of Pill Burden on Adherence, Risk of Hospitalization, and Viral Suppression in Patients with HIV Infection and AIDS Receiving Antiretroviral Therapy. Pharmacotherapy. 2016;36(4):385-401. doi:10.1002/phar.1728.

28. Guaraldi G, Menozzi M, Zona S, et al. Impact of polypharmacy on antiretroviral prescription in people living with HIV. $J$ Antimicrob Chemother. 2017;72(2):511-514. doi:10.1093/jac/dkw437.

29. Zhou S, Martin K, Corbett A, et al. Total daily pill burden in HIV-infected patients in the southern United States. AIDS Patient Care STDS. 2014;28(6):311-317. doi:10.1089/apc.2014.0010.

30. Choudhry NK, Fischer MA, Avorn J, et al. The implications of therapeutic complexity on adherence to cardiovascular medications. Arch Intern Med. 2011;171(9):814-822. http://www.ncbi.nlm.nih.gov/pubmed/2155565 9. Accessed January 24, 2018.

31. Saini SD, Schoenfeld $P$, Kaulback K, Dubinsky MC. Effect of medication dosing frequency on adherence in chronic diseases. Am J Manag Care. 2009;15(6):e22-33. http://www.ncbi.nlm.nih.gov/pubmed/1951480 
6. Accessed January 24, 2018.

32. Marcum ZA, Gellad WF. Medication Adherence to Multidrug Regimens. Clin Geriatr Med. 2012;28(2):287-300. doi:10.1016/j.cger.2012.01.008.

33. Pantuzza LL, Ceccato M das GB, Silveira MR, Junqueira LMR, Reis AMM. Association between medication regimen complexity and pharmacotherapy adherence: a systematic review. Eur J Clin Pharmacol. 2017;73(11):1475-1489. doi:10.1007/s00228017-2315-2.

34. Haro Márquez C, Cantudo Cuenca MR, Almeida González CV, Morillo Verdugo R. [Patients'adherence-related beliefs about medicines prescribed for long-term conditions in HIV patients]. Farm Hosp. 2015;39(1):2328. doi:10.7399/fh.2015.39.1.8127.

35. Borrego $Y$, Gómez-Fernández E, Jiménez R, Cantudo R, Almeida-González C V, Morillo R. Predictors of primary non-adherence to concomitant chronic treatment in HIV-infected patients with antiretroviral therapy. Eur J Hosp Pharm. February 2017:ejhpharm-2016001000. doi:10.1136/ejhpharm-2016-001000.

36. Metz KR, Fish DN, Hosokawa PW, Hirsch JD, Libby AM. Patient-Level Medication Regimen Complexity in Patients With HIV. Ann Pharmacother. 2014;48(9):1129-1137. doi: $10.1177 / 1060028014539642$.

37. Hirsch JD, Metz KR, Hosokawa PW, Libby AM. Validation of a patient-level medication regimen complexity index as a possible tool to identify patients for medication therapy management intervention. Pharmacotherapy. 2014;34(8):826-835. doi:10.1002/phar.1452.

38. Wimmer BC, Cross AJ, Jokanovic N, et al. Clinical Outcomes Associated with Medication Regimen Complexity in Older People: A Systematic Review. J Am Geriatr Soc. 2017;65(4):747-753. doi:10.1111/jgs.14682.

39. Clegg A, Young J, lliffe S, Rikkert MO, Rockwood K. Frailty in elderly people. Lancet. 2013;381(9868):752-762. doi:10.1016/S01406736(12)62167-9.

40. Guaraldi G, Brothers TD, Zona S, et al. A frailty index predicts survival and incident multimorbidity independent of markers of HIV disease severity. AIDS. 2015;29(13):16331641. doi:10.1097/QAD.0000000000000753. 